\title{
Complicações no tratamento de urolitíase devido a efeitos adversos da radioterapia pélvica
}

\author{
Urolithiasis treatment complications due to pelvic radiotherapy adverse events
}

Complicaciones en el tratamiento de la urolitiasis debido a los efectos adversos de la radioterapia pélvica

Natália Guedes Alves ${ }^{1 *}$, Rudá Mura Medeiros Paz ${ }^{2}$, Otávio Lima de Arruda Júnior ${ }^{2}$, José Miranda Rabelo Neto ${ }^{2}$, Jorge Patrick Silva da Rocha ${ }^{3}$, Pedro Henrique Athayde Pontes ${ }^{3}$, Eduardo Piotto Leonardi², Rui Wanderley Mascarenhas Júnior ${ }^{2}$.

\section{RESUMO}

Objetivo: Relatar o caso de uma paciente portadora de ureterolitíase que foi submetida à ureteroscopia, procedimento no qual ocorreram dificuldades devido ao histórico de radioterapia pélvica prévia da paciente. Detalhamento do caso: Paciente de 50 anos com história de dor lombar à direita a cerca de um ano com histórico de tratamento oncológico de braquiterapia para câncer de colo uterino. A anamnese revelou que a paciente já possuía história prévia de nefrolitíase, com cálculo de $1,6 \mathrm{~cm}$ em pelve renal direita, mas após ser submetida à Litotripsia Extracorpórea por Onda de Choque (LECO), evoluiu com uropatia obstrutiva por impactação dos fragmentos de cálculo. Esse quadro levou a paciente a realização de tratamento endourológico. A realização de ureterolitotripsia endoscópica revelou ureter com estenoses em porção distal e paredes fibrosadas, relacionadas o tratamento radioterápico prévio. Considerações finais: Nesses casos, é necessário que o urologista esteja alerta aos antecedentes clínicos de cada paciente, pois - ao se deparar com histórico positivo, terá um sinal de alerta e estará mais preparado mediante possíveis dificuldades considerando que os efeitos adversos do tratamento radioterápico podem alterar os resultados esperados tanto da LECO quando da ureterolitotripsia endoscópica, impondo dificuldades significativas ao tratamento.

Palavras-chave: Nefrolitíase, Litotripsia, Radioterapia.

\begin{abstract}
Objective: To report the case of a patient with ureterolithiasis who under went ureteroscopy, a procedure in which difficulties occurred due to the patient's previous pelvic radiotherapy. Case details: 50-year-old patient with a history of low back pain on the right for about a year with a history of oncological treatment for brachytherapy for cervical cancer. The anamnesis revealed that the patient already had a previous history of nephrolithiasis, with a $1.6 \mathrm{~cm}$ stone in the right renal pelvis, but after undergoing Shock Wave Extracorporeal Lithotripsy (ESWL), she developed obstructive uropathy due to impaction of the stone fragments. This situation led the patient to undergo endourological treatment. Endoscopic ureterolithotripsy revealed a ureter with distal stenosis and fibrous walls, related to previous radiotherapy. Final considerations: In these cases, it is necessary for the urologist to be alert to the clinical history of each patient, because - when faced with a positive history, he will have a warning sign and will be more prepared due to possible difficulties considering that the adverse effects of radiotherapy treatment may alter the expected results of both ESWL and endoscopic ureterolithotripsy, imposing significant difficulties in treatment.
\end{abstract}

Keywords: Nephrolithiasis, Lithotripsy, Radiotherapy.

1 Universidade do Estado do Pará (UEPA). Belém - PA.

*E-mail: natyguedes31@gmail.com

${ }^{2}$ Fundação Hospital de Clínicas Gaspar Vianna (FHCGV). Belém - PA.

${ }^{3}$ Universidade Federal do Pará (UFPA). Belém - PA. 


\section{RESUMEN}

Objetivo: informar el caso de un paciente com ureterolitiasis que se sometió a ureteroscopia, um procedimento en el que se produjeron dificultades debido a la radioterapia pélvica previa del paciente. Detalles del caso: paciente de 50 años con antecedentes de dolor lumbar a la derecha durante aproximadamente um año con antecedentes de tratamiento oncológico para braquiterapia para el cáncer cervical. La anamnesis reveló que la paciente ya tenía antecedentes de nefrolitiasis, con un cálculo de $1,6 \mathrm{~cm}$ em la pelvis renal derecha, pero después de someterse a lalitotricia extracorpórea por ondas de choque (ESWL), desarrolló una uropatía obstructiva debido a la impactación de los fragmentos de cálculos. Esta situación llevó al paciente a someterse a um tratamento endourológico. La ureterolitotripsia endoscópica reveló um ureter com estenosis distal y paredes fibrosas, relacionado con radioterapia previa. Consideraciones finales: en estos casos, es necesario que el urólogo esté alerta a la historia clínica de cada paciente, ya que, cuando se enfrenta a una historia positiva, tendrá una señal de advertencia y estará más preparado debido a posibles dificultades teniendo en cuenta que los efectos adversos del tratamento con radioterapia pueden alterar los resultados esperados tanto de ESWL como de ureterolitotripsia endoscópica, imponiendo dificultades significativas en el tratamiento.

Palabras clave: Nefrolitiasis, Litotricia, Radioterapia.

\section{INTRODUÇÃO}

As neoplasias pélvicas englobam os canceres de útero, ovários, bexiga, próstata, reto e de colo de útero. O Câncer de colo de útero é a quarta neoplasia mais incidente em mulheres. Estima-se que, anualmente,530.000 novos casos sejam diagnosticados mundialmente e 270.000 mortes decorrentes da doenças (SMALL W, et al., 2017). Diante dos números relacionados à prevalência e à mortalidade desta neoplasia, diversos avanços relacionados à prevenção, ao diagnóstico e ao tratamento ocorreram nos últimos anos, melhorando significativamente o manejo da doença.

O tratamento está diretamente relacionado ao estadiamento do câncer. Existem diversas formas de manejar a neoplasia de colo uterino, o que irá determinar a escolha da terapêutica são os fatores relacionados ao paciente quanto ao estadiamento do tumor, podendo variar entre abordagem cirúrgica, radioterapia, braquiterapia e quimioterapia (KISSEL M, et al., 2020). Uma das modalidades mais eficazes contra neoplasia de colo de útero é a combinação de radioterapia externa e braquiterapia intracavitária (LIU R, et al., 2015), cujos resultados mostram-se com baixa toxicidade e boa resposta no tratamento de tumores descobertos precocemente (VORDERMARK D, 2016). Porém, tais métodos afetam também as células sadias do indivíduo, principalmente as células pertencentes a tecidos próximos anatomicamente do órgão alvo, causando injúria celular (YAMADA T, et al., 2018).

Acerca dos efeitos colaterais da radioterapia, estes têm como principal mecanismo os danos sobre o material genético, assim, afetando a divisão celular e gerando erros na replicação. $O$ dano tecidual induzido pela radioterapia afeta principalmente os vasos sanguíneos, propiciando eventos como oclusão, trombose e neovascularização; ocorre também a atrofia e contração do tecido devido ao aumento da proliferação de fibroblastos. Assim, as principais complicações decorrentes da radioterapia no trato urinário são ureterite actínica, fístula vesico-vaginal, cistite actínica e hematúria. Geralmente, esses efeitos colaterais surgem após dois anos de tratamento, porém mesmo após 100 meses de tratamento, há possibilidades elevadas de surgimento de efeitos adversos (YAMADA T, et al., 2018).

A nefrolitíase atinge de $7 \%$ a $13 \%$ da população norte americana. Observa-se também que desde a década de 80, as taxas de urolitíase têm crescido consideravelmente entre adultos. Quanto ao Brasil, apenas no ano de 2010, foram admitidos cerca de 70.000 pacientes por conta de calculose urinária dentro do Sistema Único de Saúde.

No país, também é notório o aumento de $69 \%$ nas admissões por urolitíase de 1996 à 2010. (SOROKIN I, et al., 2016). Assim, houve desenvolvimento de técnicas minimamente invasivas para tratar dessa patologia. Dando ênfase para a ureterolitotripsia endoscópica e para a LECO, ambos são procedimentos seguros, com baixo risco de complicações e que possuem resultados excelentes quando indicadas adequadamente, 
podendo culminar com pacientes alcançado o estágio livre de cálculos. A indicação e o sucesso do procedimento estão diretamente relacionados à localização e ao tamanho do cálculo. Apesar de serem técnicas minimamente invasivas, podem apresentar algumas complicações. Condições anatômicas desfavoráveis, como estenoses ureterais, podem aumentar a probabilidade de dificuldades intraoperatórias e pós operatórias (SOMANI BK, et al., 2016). Dessa forma, os possíveis efeitos adversos no trato urinário gerados pelo tratamento radioterápico, principalmente quando realizado em região pélvica, podem dificultar fases importantes do tratamento da urolitíase, tornando o manejo desses pacientes desafiador ao urologista.

Este artigo científico tem como objetivo relatar o caso de uma paciente com histórico de tratamento radioterápico para câncer de colo uterino que foi submetida a LECO e, posteriormente, à ureterolitotripsia endoscópica, apresentando complicações não esperadas durante o manejo do caso.

\section{DETALHAMENTO DO CASO}

Paciente do sexo feminino, 50 anos, solteira, parda, residente em Belém, Pará, atendida no ambulatório de Urologia, em junho de 2018, com história de dor lombar à direita com irradiação para flanco direito e membros inferiores de início a cerca de um ano. Nega febre, outros sintomas miccionais ou comorbidades.

Quanto aos antecedentes mórbidos e pessoais, em 2016, a paciente foi diagnosticada com carcinoma epidermóide do colo uterino grau III, havendo disseminação da neoplasia para porção inferior da vagina e paredes da pelve. Foi submetida ao tratamento oncológico por dois anos, com realização de braquiterapia para câncer de colo uterino de alta taxa de dose, com dose de 2800cGy em 4 frações, utilizando sonda e anel; além de sessões de quimioterapia no ano de 2017. Atualmente, faz seguimento ambulatorial oncológico de rotina, não apresentando metástases.

Como antecedentes, possuía história prévia de nefrolitíase, com cálculo de 1,6cm em pelve renal direita. Foi submetida à LECO, porém permaneceu com sintomas álgicos. Na reavaliação da paciente, foi solicitada uma tomografia computadorizada, a qual exibiu numerosos cálculos no interior dos terços médio e distal do ureter direito, determinando acentuada uropatia obstrutiva, com hidronefrose e rua de cálculos (Figura 1). Assim, a paciente foi encaminhada para realização de tratamento endourológico.

Figura 1 - Tomografia computadorizada pré-operatória evidenciando hidronefrose acentuada em rim direito e rua de cálculos em ureter distal direito.

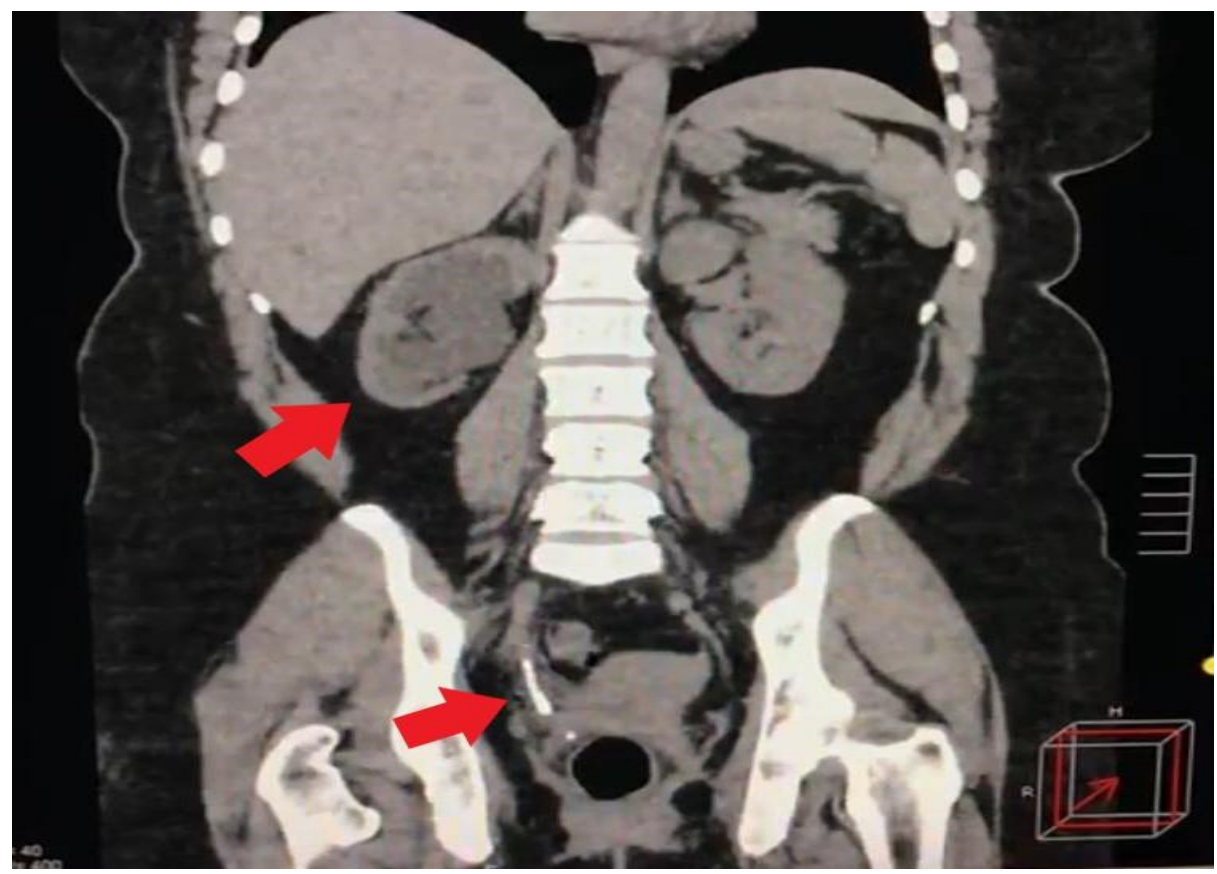

Fonte: Alves NG, et al., 2020. 
Foi admitida para realização de ureterolitotripsia endoscópica à direita em março de 2019. Inicialmente submetida a uma cistoscopia e passagem de fio guia hidrofílico em ureter direito, com parada da progressão do fio guia em ureter distal. Ureteroscopia evidenciou parede ureteral de aspecto actínico com cálculos impactados. Realizada a ureterolitotripsia e remoção de fragmentos de cálculos, foi então tentada novamente a passagem do fio guia hidrofílico sem sucesso, devido à estenose ureteral e rua de cálculos. Então, optouse pela conversão do procedimento à ureterolitotomia aberta. Realizou-se uma incisão de Gibson à direita, diérese por planos e identificação do ureter direito, o qual encontrava-se dilatado, com superfície rígida, irregular e aderido às estruturas abdominais adjacentes. Fez-se a ureterotomia e a exérese de fragmentos de cálculos. Foi realizada a passagem do fio guia hidrofílico até o rim direito sem dificuldades, porém durante a tentativa de passagem do fio até a bexiga, não foi possível, devido às aderências no local, à estenose do ureter distal e aos cálculos. Então, foi realizado reimplante ureteral após passagem do cateter duplo jota até a bexiga, sob técnica de Lich-Gregorior e bexiga psoica (Figura 2). Foi então realizada a drenagem laminar, revisão da cavidade e síntese por planos.

No pós-operatório imediato, paciente foi encaminhada para leito de UTI, apresentava-se consciente e orientada, hemodinamicamente estável sem uso de drogas vasoativas. Ao exame físico, abdomen flácido, doloroso à palpação em baixo ventre e ruídos hidroaéreos audíveis, porém reduzidos. Diurése reduzida com aspect concentrado $(50 \mathrm{ml} / 6 \mathrm{~h})$. Foi iniciada antibioticoterapia com Cefepime. No segundo dia, paciente apresentou-se estável, afebril, com aumento progressivo da diurése até atingir os padrões normais no 3으 dia pós-operatório. A ferida operatória permaneceu limpa e seca, evoluindo satisfatoriamente no processo de cicatrização. Não foi observado débito no dreno laminar. Quanto aos níveis de ureia e creatinina, elevaramse no primeiro dia do pós-operatório, mas voltaram aos padrões de normalidade após o segundo dia de pósoperatório.

Foi dada a alta hospitalar após uma semana de internação, período utilizado para observar se a paciente evoluiria com alguma complicação, como fistula urinária, o que não ocorreu e culminou com bom prognóstico. Um mês depois, foi feita a retirada do cateter duplo J. Após 3 meses, paciente realizou tomografia computadorizada de controle, a qual não exibiu sinais obstrutivos ou cálculos no trato urinário. Seis meses após o procedimento, paciente evolui sem queixas urinárias.

Figura 2 - Tomografia computadorizada após 3 meses do procedimento cirúrgico evidenciando a bexiga psoica (setas)

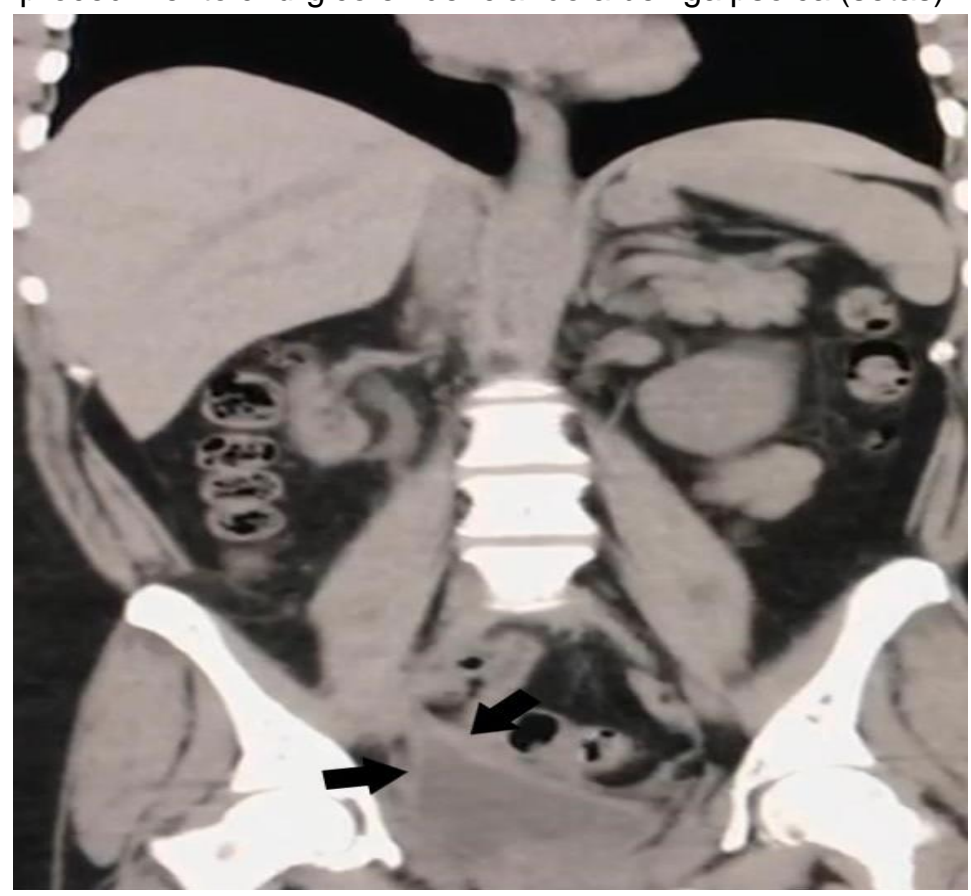

Fonte: Alves NG, et al., 2020. 


\section{DISCUSSÃO}

A ureterolitotripsia é um método que tem demonstrado avanços significativos nas últimas décadas, com destaque para a realização destes procedimentos por via endourológica, o que - além de aumentar a eficácia - contribuiu para maior segurança durante a realização do procedimento e a melhor recuperação do paciente durante o pós-operatório (ALENEZI H e DENSTEDT J, 2015). Uma das características da endourologia aplicada à litotripsia é a mínima invasividade, o que propicia uma recuperação mais rápida e menor risco de intercorrências ao paciente. Entretanto, ainda existem complicações atreladas à ureterolitotripsia endoscópica (D'ADDESSI A e BASSI P, 2011).

No caso relatado, a paciente havia sido submetida à tratamento radioterápico para câncer de colo uterino dois anos antes do tratamento para nefrolitíase. Toia B, et al. (2018) afirma que o tratamento radioterápico específico para câncer de colo uterino é o principal causador de efeitos adversos quando comparado aos tratamentos para cânceres pélvicos em outros órgãos, como a próstata. É evidente que a radioterapia e a braquiterapia são fundamentais no manejo eficaz dessa neoplasia, sendo as práticas mais adotadas mundialmente. Porém, a toxicidade celular desta terapêutica atinge também tecido sadio, afetando-o negativamente. Ao serem analisados os casos que evoluíram com complicações, observa-se que as lesões mais comuns são a estenose ureteral, fístula vesico-vaginal e hematúria, as quais desenvolvem-se após 2 anos de tratamento, em média (LIBERMAN D, et al., 2014).

Quanto à estenose ureteral pós radioterapia, esta é de difícil manejo e dificulta a realização de procedimentos por via endourológica (LIBERMAN D, et al., 2014). Lobo N, et al. (2018) relatou a ocorrência de estenoses principalmente em ureter distal, devido à própria anatomia local, haja vista que o ponto de inserção dos ureteres na bexiga está próximo do colo uterino e, dessa forma, mais exposto ao dano radioterápico, o que condiz com o caso apresentado. Também foi percebido durante o ato cirúrgico a existência de estenoses em ureter distal, próximo à junção vesico-ureteral, o que condiz com a localização de lesão pós-radioterapia pélvica (PAPADOPOULOU I, et al., 2016).

Ademais, a presença das estenoses no ureter distal da paciente dificultou a excreção dos fragmentos de cálculo pós-LECO, haja vista que - com a redução do diâmetro da luz ureteral - há maior probabilidade de impactação de cálculos. Este fato foi observado na evolução da paciente e culminou com quadro obstrutivo, gerando as dores lombares informadas na anamnese. Essas alterações pós-radioterápicas também aumentam a probabilidade de ocorrência de complicações durante ureterolitotripsia (LEGEMATE JD, et al., 2017). No caso, foi observada dificuldade para passagem do fio guia hidrofílico no ureter distal, portanto, impossibilitando a realização segura do procedimento. Tal fato concorda com o que é afirmado por D'Addessi A e Bassi P (2011), os quais reiteram que a ureterite pode dificultar o intraoperatório, principalmente durante passagem do ureteroscópio no ureter intramural para alcançar o cálculo.

Também é importante salientar que após a conversão do procedimento para ureterolitotomia aberta, foi observando ureter médio dilatado, ureter distal com superfície rígida, irregular e aderido às estruturas abdominais adjacentes; características atípicas da parede ureteral. Estas podem ser atribuídas a consequências da radioterapia (LIBERMAN D, et al., 2014), pois este tipo de tratamento, ao atingir o urotélio, aumenta a proliferação de fibroblastos e, consequentemente, ocorre a fibrose das paredes afetadas, alterando sua morfologia (LIBERMAN D, et al., 2014). No caso relatado, foi realizado um reimplante ureteral, o qual, na maioria dos casos, não é viável devido à isquemia do segmento distal (ALENEZI H e DENSTEDT J, 2015). Porém, foi realizada a retirada da porção fibrótica do ureter e, então, foi possível à realização do reimplante ureteral após passagem do cateter duplo jota até a bexiga, sob técnica de Lich-Gregorior e bexiga psoica.

Devido à realização da radioterapia pélvica prévia associada à ureterolitotripsia com ureterotomia e reimplante ureteral, no pós operatório, houve maior atenção para a possibilidade de fístula urinária.

Segundo Machida $\mathrm{H}$, et al. (2019), a radioterapia pélvica utilizada no tratamento oncológico aumenta a probabilidade de fístula urinária. Dessa forma, a paciente foi mantida em observação durante 7 dias para afastar essa possibilidade. Tal complicação não ocorreu e culminou em bom prognóstico da paciente, que recebeu alta hospitalar. 
Dessa forma, frente à alta prevalência de câncer de colo de útero em mulheres no mundo inteiro em conjunto aos números crescentes de urolitíase na população, deve-se observar os desafios atrelados ao tratamento de pacientes com história prévia de radioterapia pélvica. Assim, é necessário que o urologista adote cuidados adicionais na abordagem, anamnese e indicação da terapêutica a ser adotada, conforme os antecedentes de cada indivíduo, pois, embora a LECO e a ureterolitotripsia endoscópica sejam procedimentos resolutivos e de baixo potencial de complicações, os efeitos adversos ao tratamento radioterápico podem alterar os resultados esperados para os respectivos procedimentos, impondo dificuldades significativas tanto durante a excreção dos fragmentos de cálculo pós-LECO quanto durante a realização da ureterolitotripsia endoscópica.

No presente artigo, foi relatado o caso de uma paciente atendida para tratamento de nefrolitíase à direita. Esta possuía histórico prévio de radioterapia pélvica há 2 anos. Em seu manejo, primeiramente, foi realizada a LECO, porém a paciente evoluiu com rua de cálculos e obstrução em ureter direito. Então, foi realizada à ureterolitotripsia endoscópica, que foi impossibilitada pelas alterações actínicas do ureter, sendo convertida para ureterotomia aberta associada à reimplante ureteral. Concluí-se que tais complicações, não esperadas durante a realização desses procedimentos, têm relação com o histórico de radioterapia pélvica da paciente, devendo o urologista adotar cuidados adicionais no manejo desses pacientes. Assim, caso se depare com um histórico positivo, estará preparado para agir mediante possíveis dificuldades.

\section{REFERÊNCIAS}

1. ALENEZI H, DENSTEDT J. Flexible ureteroscopy: Technological advancements, current indications and outcomes in the treatment of urolithiasis. Asian Journal of Urology, 2015; 2: 133-141.

2. D'ADDESSI A e BASSI P. Ureterorenoscopy: Avoiding and Managing the Complications. Urologia Internationalis, 2011; 87: 251-259.

3. KISSEL M, et al. Challenges and advances in cervix cancer treatment in elder women. Cancer Treatment Reviews, 2020; 84: 101976.

4. LIU R, et al. High dose rate versus low dose rate intracavity brachytherapy for locally advanced uterine cérvix cancer. Cochrane Database of Systematic Reviews, 2015.

5. LIBERMAN D, et al. Urinary adverse effects of pelvic radiotherapy. Translational Andrology and Urology, 2014; 3 : 186-195.

6. LEGEMATE JD, et al. Characteristics and outcomes of ureteroscopic treatment in 2650 patients with impacted ureteral stones. World Journal of Urology, 2017; 35: 1497-1506.

7. LOBO N, et al. Urologic complications following pelvic radiotherapy. Urology, 2018; 122: 1-9.

8. MACHIDA H, et al. Profile of treatment-related complications in women with clinical stage IB-IIB cervical cancer: A nationwide cohort study in Japan. PLos ONE, 2019; 14: 1-15.

9. PAPADOPOULOU I, et al. Post-Radiation Therapy Imaging Appearances in Cervical Carcinoma. Radiographics, 2016; 36: 538-553.

10. SMALL W, et al. Cervical cancer: a global healthcrisis. Cancer. 2017; 13:2404-2412.

11. SOMANI BK, et al. Complications associated with ureterorenoscopy (URS) related to treatment of urolithiasis: the Clinical Research Office of Endourological Society URS Global study. World Journal of Urology, 2016; 35: 675-681.

12. SOROKIN I, et al: Epidemiology of stone disease across the world. World Journal of Urology, 2017; 35: 1301-1320.

13. TOIA B, et al. Outcomes of reconstructive urinary tract surgery after pelvic radiotherapy. Scandinavian Journal of Urology, 2019; 53: 156-160.

14. VORDERMARK D. Radiotherapy of cervical cancer. Oncology Research and Treatment, 2016; 39: 516-520.

15. YAMADA T, et al. Analysis of late adverse events and their chronological changes after radiation therapy for cervical cancer. Nagoya Journal Medical Science, 2018; 80: 487-496. 